\author{
(c) (i) (8) \\ Journal Terapan Manajemen dan Bisnis is licensed under \\ A Creative Commons Attribution-NonCommercial 4.0 International License
}

\title{
THE EFFECT OF ENTREPRENEURSHIP LEADERSHIP AND MOTIVATION ON EMPLOYEES' SATISFACTION AT SERANG RAYA UNIVERSITY
}

\author{
Hamdan \\ Serang Raya University, Indonesia \\ E-mail: hamdanunsera@gmail.com
}

\begin{abstract}
A leader who has an entrepreneurial spirit or entrepreneurial leadership and motivation is absolutely necessary as an intangible culture, a non-physical structural capability that is able to move a physical figure, dare to take risks and prioritize work performance. so this study aims to determine the Effect of Entrepreneurship Leadership and Motivation on Employee Job Satisfaction at Serang Raya University. This study uses a descriptive method and in the form of a questionnaire distributed to 77 respondents who were the samples of this study, the data came from populations that were normally distributed and had the same variance. The technique of collecting data using a questionnaire with a Likert scale measurement. correlation coefficient $\mathrm{R}=0.723$, while the coefficient of determination seen from $\mathrm{R}$ Square $=0.522$ which means that entrepreneurial leadership and motivation of independent variables in explaining the dependent variable by $52.2 \%$ the remaining $47.8 \%$ is influenced by other factors such as work culture, employee discipline, environment work, etc. which were not included in this study. And there is a significant influence between entrepreneurial leadership and motivation on employee job satisfaction at Serang Raya University in the $t$ test, the results obtained $t$ count $>t$ table $(4,281>1,992)$, it can be concluded that there is a significant influence between entrepreneurial leadership $\left(X_{1}\right)$ and motivation $\left(X_{2}\right)$ on employee job satisfaction (Y) at Serang Raya University.
\end{abstract}

Keywords: Entrepreneurship Leadership; Motivation; Intangible Culture; Job Satisfaction; Entrepreneurship Soul

\section{Introduction}

Each organization, a leader who has an entrepreneurial spirit is one of the main factors in supporting the success of an organization to achieve goals, success or effectiveness of leadership is not only measured how to empower subordinates but also the ability to run policies through leadership style or style, leadership style depends on individual leaders who have an entrepreneurial spirit in moving subordinates based on their function as superiors. The leadership role that is very strategic and important for achieving the vision, mission, and goals of the organization, is one motive that encourages people to always investigate the intricacies related to leadership. Entrepreneurship is a process in doing something new or creative and different (innovative) that is useful in providing more value. In other words, entrepreneurship is a courage to make efforts to fulfill the needs of life carried out by someone on the basis of ability by utilizing everything the potential possessed to produce something that is beneficial to himself and others, someone is considered an entrepreneur if 
he has fulfilled the definition of entrepreneurship itself. The leader of good entrepreneurship is a leader who is always forward-oriented in leading his employees well. Entrepreneurs have different leadership styles, they develop their own leadership style in accordance with their personal character in advancing.

Leadership at the University of Serang Raya fully implements leadership functions such as instructive functions that have not been fully implemented, communication of superiors with subordinates who are not well established, participation of superiors to subordinates who are still low, delegation functions that have not been fully implemented so that decision making cannot be carried out quickly, there was still a lack of direction, coordination and supervision from the leadership. Because the leader has not fully carried out the leadership functions properly. Motivation is defined by several researchers as a measure of the strength of identity and involvement of employees in organizational goals and values. Motivation is obtained as a better indicator of "leavers" and "stayers" than job satisfaction. Another study found that job satisfaction is related to the task environment, while motivation is related to achievement in organizational empowerment. With the motivation given, it is expected that employee job satisfaction will increase, defining motivation as an attitude that reflects employee loyalty to the organization and is an ongoing process where members of the organization express their concern for the organization, the success of the organization and continuous progress.

According to Tangkilisan (2005: 78) leadership in an organization or field of work has a different function between an organization with one another, the function of the leader basically depends on several things consisting of social situations in the organization, characteristics of leaders and the number of members. this study discusses the main functions that a leader must have. According to Siagian (1989) regarding the understanding of techniques and ways to measure the level of job satisfaction of employees, where job satisfaction is the emotional attitude of employees who love and are happy with the work they carry, where this attitude is reflected by work morale, discipline and work performance of each employee. Job satisfaction is a feeling of joy and gives a positive value to an assessment of a job.

The level of job satisfaction of employees at the University of Serang Raya such as fulfilling the needs of life that have not been adequately fulfilled due to minimum wages that are still small so that the level of individual satisfaction has not been achieved.

\section{Literature Review}

According to Tangkilisan (2005: 78) Leadership has a function in a working group with another working group. This is because leaders have functions in an organization, social situations in the organization, characteristics of leaders in a work group. Furthermore Tangkilisan (2005: 30) explains that there are some of the most important functions in carrying out a task by leaders as berikuy: establishing, organizing, motivating and communicating, evaluating, and developing subordinates including himself. Therefore, a leader functions to prepare resources reliable so that it requires a support and motivation from members.

Successful leadership will be able to provide direction and give examples to all employees to achieve results and objectives to be achieved. Because leaders must be able to provide examples in terms of all things, such as prioritizing the interests of shared goals above personal interests. In addition, leaders are able to communicate with all parties, so that good communication can be established between all employees. Leaders also in the decision-making process must really see from all aspects. That leader must also have superior personal qualities than his subordinates, besides that leaders must be able to give encouragement to their subordinates to advance. 


\section{Research Methods}

Data Processing Method. This study will use a survey method with a quantitative approach using data analysis techniques using Likert scale consisting of testing validity, reliability testing, and classic assumption tests. Normality Test, Linearity Test, Heterocedasticity Test, Interpretation Average Analysis, Coefficient of Determination Analysis, Regression Analysis, Test Hypothesis (Test "t"), Test F.

\section{Results and Discussion}

A. Instrument Test

Validity test

Table 1 Results of Test Validity of Leadership Variables (X1) Item-Total Statistics

\begin{tabular}{|c|c|c|c|c|c|}
\hline & $\begin{array}{l}\text { Scale Mean } \\
\text { if Item } \\
\text { Deleted }\end{array}$ & $\begin{array}{c}\text { Scale } \\
\text { Variance if } \\
\text { Item } \\
\text { Deleted } \\
\end{array}$ & $\begin{array}{l}\text { Corrected } \\
\text { Item-Total } \\
\text { Correlation }\end{array}$ & $\begin{array}{c}\text { Squared } \\
\text { Multiple } \\
\text { Correlation }\end{array}$ & $\begin{array}{l}\text { Cronbach's } \\
\text { Alpha if } \\
\text { Item } \\
\text { Deleted } \\
\end{array}$ \\
\hline $\mathrm{X} 1.1$ & 42.00 & 5.526 & .380 & .326 & .728 \\
\hline $\mathrm{X} 1.2$ & 42.05 & 5.024 & .608 & .742 & .692 \\
\hline$X 1.3$ & 42.05 & 5.366 & .437 & .603 & .720 \\
\hline X1.4 & 42.01 & 5.776 & .257 & .570 & .746 \\
\hline X1.5 & 42.14 & 5.203 & .496 & .525 & .710 \\
\hline X1.6 & 41.91 & 5.610 & .396 & .375 & .726 \\
\hline X1.7 & 41.92 & 5.415 & .489 & .404 & .713 \\
\hline X1.8 & 42.13 & 5.351 & .426 & .577 & .722 \\
\hline X1.9 & 42.00 & 5.816 & .243 & .460 & .748 \\
\hline $\mathrm{X} 1.10$ & 41.86 & 5.808 & .338 & .278 & .734 \\
\hline Number & & $r_{\text {count }}$ & rtable & & Status \\
\hline 1 & & 0.380 & 0.224 & & Valid \\
\hline 2 & & 0.608 & 0.224 & & Valid \\
\hline 3 & & 0.437 & 0.224 & & Valid \\
\hline 4 & & 0.257 & 0.224 & & Valid \\
\hline 5 & & 0.496 & 0.224 & & Valid \\
\hline 6 & & 0.396 & 0.224 & & Valid \\
\hline 7 & & 0.489 & 0.224 & & Valid \\
\hline 8 & & 0.426 & 0.224 & & Valid \\
\hline 9 & & 0.243 & 0.224 & & Valid \\
\hline 10 & & 0.338 & 0.224 & & Valid \\
\hline
\end{tabular}

Table 2 Results of Test Validity of Motivation Variables (X2) Item-Total Statistics

\begin{tabular}{lrrrrr}
\hline & $\begin{array}{c}\text { Scale } \\
\text { Mean if } \\
\text { Item } \\
\text { Deleted }\end{array}$ & $\begin{array}{c}\text { Scale } \\
\text { Variance if } \\
\text { Item } \\
\text { Deleted }\end{array}$ & $\begin{array}{c}\text { Corrected } \\
\text { Item-Total } \\
\text { Correlation }\end{array}$ & $\begin{array}{c}\text { Squared } \\
\text { Multiple } \\
\text { Correlation }\end{array}$ & $\begin{array}{c}\text { Cronbach's } \\
\text { Alpha if Item } \\
\text { Deleted }\end{array}$ \\
\hline X2.1 & 40.10 & 14.884 & .241 & .143 & .692 \\
X2.2 & 40.38 & 12.817 & .505 & .513 & .644 \\
X2.3 & 40.16 & 13.554 & .424 & .510 & .662 \\
\hline
\end{tabular}


Jurnal Terapan Manajemen dan Bisnis

Volume 4 Number 2 September 2018. Page 171-183

e-ISSN: 2477-5282 p-ISSN: 2599-3127

\begin{tabular}{|c|c|c|c|c|c|}
\hline & $\begin{array}{c}\text { Scale } \\
\text { Mean if } \\
\text { Item } \\
\text { Deleted }\end{array}$ & $\begin{array}{c}\text { Scale } \\
\text { Variance if } \\
\text { Item } \\
\text { Deleted }\end{array}$ & $\begin{array}{c}\text { Corrected } \\
\text { Item-Total } \\
\text { Correlation }\end{array}$ & $\begin{array}{l}\text { Squared } \\
\text { Multiple } \\
\text { Correlation }\end{array}$ & $\begin{array}{c}\text { Cronbach's } \\
\text { Alpha if Item } \\
\text { Deleted }\end{array}$ \\
\hline$X 2.4$ & 40.43 & 13.301 & .365 & .663 & .674 \\
\hline$X 2.5$ & 40.21 & 13.877 & .582 & .493 & .647 \\
\hline$X 2.6$ & 40.18 & 14.703 & .248 & .531 & .692 \\
\hline$X 2.7$ & 40.32 & 12.959 & .438 & .522 & .658 \\
\hline$X 2.8$ & 40.62 & 13.633 & .287 & .329 & .692 \\
\hline X2.9 & 40.05 & 14.918 & .265 & .574 & .688 \\
\hline$X 2.10$ & 39.97 & 15.236 & .293 & .456 & .685 \\
\hline Number & & rount & rtable & & Status \\
\hline 1 & & 0.241 & 0.224 & & Valid \\
\hline 2 & & 0.505 & 0.224 & & Valid \\
\hline 3 & & 0.424 & 0.224 & & Valid \\
\hline 4 & & 0.365 & 0.224 & & Valid \\
\hline 5 & & 0.582 & 0.224 & & Valid \\
\hline 6 & & 0.248 & 0.224 & & Valid \\
\hline 7 & & 0.438 & 0.224 & & Valid \\
\hline 8 & & 0.287 & 0.224 & & Valid \\
\hline 9 & & 0.265 & 0.224 & & Valid \\
\hline 10 & & 0.293 & 0.224 & & Valid \\
\hline
\end{tabular}

Table 3 Results of Variable Validity Test for Job Satisfaction (Y) Item-Total Statistics

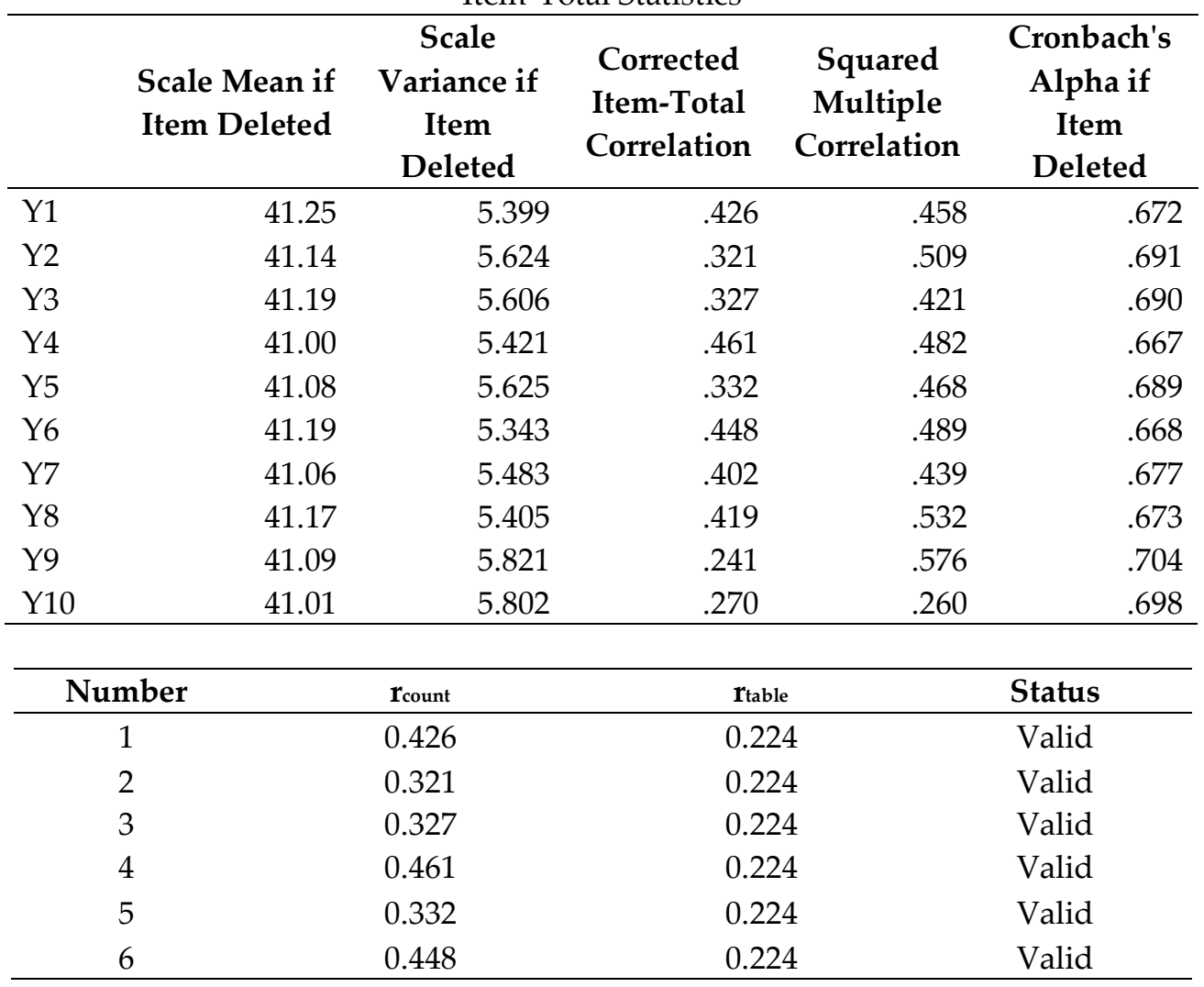




\begin{tabular}{cccc}
\hline Number & rcount & rtable & Status \\
\hline 7 & 0.402 & 0.224 & Valid \\
8 & 0.419 & 0.224 & Valid \\
9 & 0.241 & 0.224 & Valid \\
10 & 0.270 & 0.224 & Valid \\
\hline
\end{tabular}

Reliability Test

\begin{tabular}{rrrr} 
Table 4 Variable Reliability Test Results for Entrepreneurship Leadership (X1) \\
\hline Cronbach's Alpha & $\begin{array}{c}\text { Cronbach's Alpha Based on } \\
\text { Standardized Items }\end{array}$ & N of Items & \\
\hline .745 & & .744 & 10 \\
\hline
\end{tabular}

Table 5 Motivation Variable Reliability Test Results (X2)

\begin{tabular}{cccc}
\hline $\begin{array}{c}\text { Cronbach's } \\
\text { Alpha }\end{array}$ & $\begin{array}{c}\text { Cronbach's Alpha Based on } \\
\text { Standardized Items }\end{array}$ & N of Items & \\
\hline .697 & & .706 & 10 \\
\hline
\end{tabular}

Table 6 Variable Reliability Tests for Job Satisfaction (Y)

\begin{tabular}{rrrr}
\hline Cronbach's Alpha & $\begin{array}{c}\text { Cronbach's Alpha Based on } \\
\text { Standardized Items }\end{array}$ & N of Items \\
\hline .706 & & .706 & 10 \\
\hline
\end{tabular}

Table 7 Comparison of Alpha Values with rTable

\begin{tabular}{cccc}
\hline Variable & Cronbach's Alpha & $\begin{array}{c}\text { rtable } \\
(\boldsymbol{\alpha} ; \mathbf{0 . 0 5 .} \mathbf{d f} \mathbf{7 7})\end{array}$ & Status \\
\hline Leadership & 0.745 & 0.600 & Reliable \\
Motivation & 0.697 & 0.600 & Reliable \\
Job Satisfaction & 0.706 & 0.600 & Reliable \\
\hline
\end{tabular}

B. Test of Classical Assumptions

Normality test

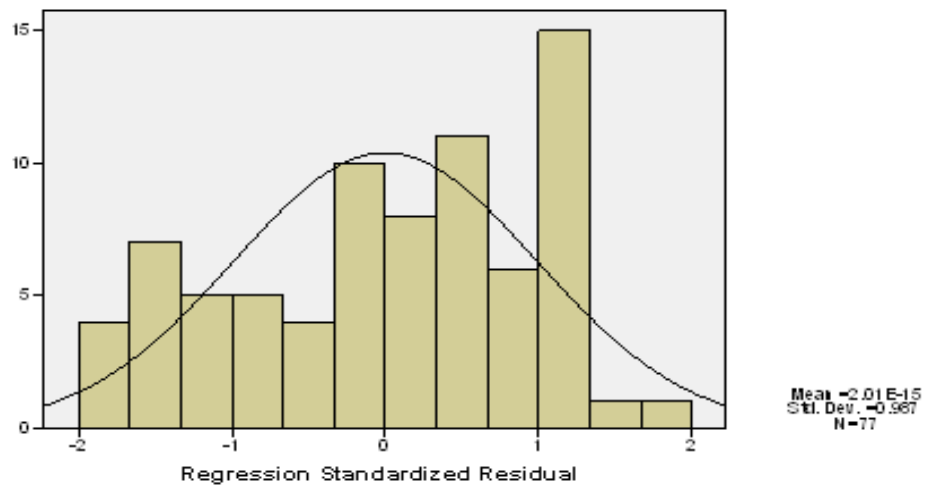

Fig. 1 Variable Histogram of Job Satisfaction 
Normal PfP Plot of Regression Standardized Residual

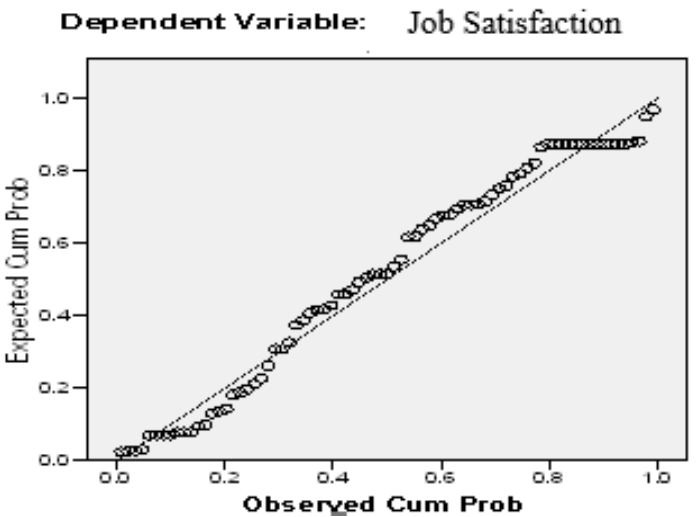

Fig. 2 Normal P-P of Regression

Multicollinearity Test

Table 8 Multicollinearity Coefficients ${ }^{a}$

\begin{tabular}{llrr}
\hline \multirow{2}{*}{ Model } & \multicolumn{2}{c}{ Collinearity Statistics } \\
\cline { 3 - 4 } & & Tolerance & VIF \\
\hline 1 & Leadership & .854 & 1.171 \\
& Motivation & .854 & 1.171 \\
\hline
\end{tabular}

a. Dependent Variable: Job Satisfaction

From the coefficient table above shows that the VIF value of the variables $\mathrm{X} 1$ and $\mathrm{X} 2$ above is 1.171 , which means $<10$, judging from the tolerance value, the tolerance value of the two variables approaches number 1 .

Heteroscedasticity Test

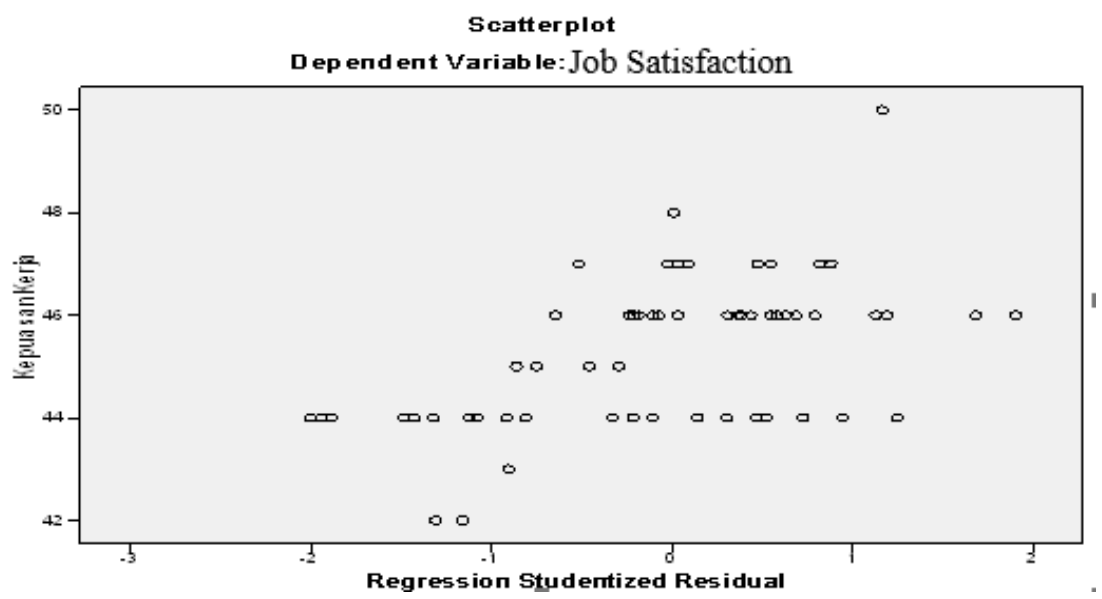

Fig. 3 Scatterplot 
Test Autocorrelation

Table 9 Autocorrelation Test Model Summary (b)

\begin{tabular}{rrrrrr}
\hline Model & R & R Square & $\begin{array}{l}\text { Adjusted } \\
\text { R Square }\end{array}$ & $\begin{array}{l}\text { Std. Error of } \\
\text { the Estimate }\end{array}$ & $\begin{array}{l}\text { Durbin- } \\
\text { Watson }\end{array}$ \\
\hline 1 & $.723(\mathrm{a})$ & .522 & .509 & 1.620 & 1.280 \\
\hline
\end{tabular}

a Predictors: (Constant), Leadership, Motivation

b Dependent Variable: Employee performance

C. Hypothesis Test

The Effect of Entrepreneurship Leadership (X1) on Job Satisfaction (Y) Regression Test.

Table 10 Multiple Linear Regression Coefficients ${ }^{a}$

\begin{tabular}{|c|c|c|c|c|c|c|}
\hline \multirow{2}{*}{\multicolumn{2}{|c|}{ Model }} & \multicolumn{2}{|c|}{$\begin{array}{c}\text { Unstandardized } \\
\text { Coefficients }\end{array}$} & \multirow{2}{*}{$\begin{array}{c}\begin{array}{c}\text { Standardized } \\
\text { Coefficients }\end{array} \\
\text { Beta }\end{array}$} & \multirow{2}{*}{$\begin{array}{l}\mathbf{T} \\
\mathbf{B}\end{array}$} & \multirow{2}{*}{$\begin{array}{c}\text { Sig. } \\
\text { Std. } \\
\text { Error }\end{array}$} \\
\hline & & B & $\begin{array}{l}\text { Std. } \\
\text { Error }\end{array}$ & & & \\
\hline \multirow[t]{2}{*}{1} & (Constant) & 15,742 & 3,386 & & 4,649 & ,000 \\
\hline & $\begin{array}{l}\text { Leadership } \\
\text { Entrepreneurship }\end{array}$ & ,646 & ,072 & ,718 & 8,922 & ,000 \\
\hline
\end{tabular}

Dependent Variable: Job Satisfaction

Y : Entrepreneurship leadership (X1)

a $: 15.742$

$\mathrm{bX}_{2} \quad: 0.646$

So that it is expressed in the form of a regression equation as follows:

$\mathrm{Y}=\mathrm{a}+\mathrm{b} \mathrm{X}_{1}$

$\mathrm{Y}=15,742+0.646 \mathrm{X} 1$

From the results of the regression equation above shows if entrepreneurial leadership (X1) value is 0 (zero) then job satisfaction $(\mathrm{Y})$ is 15,742 While entrepreneurial leadership regression coefficient (X1) of 0.646 indicates that each addition of one unit of entrepreneurial leadership will increase job satisfaction by 15,742 .

Coefficient of Determination (KD)

Table 11 Correlation Coefficients Between Entrepreneurship Leadership Against Job Satisfaction

\begin{tabular}{llrrr}
\hline Model & $\mathbf{R}$ & $\begin{array}{c}\mathbf{R} \\
\text { Square }\end{array}$ & $\begin{array}{c}\text { Adjusted } \mathbf{R} \\
\text { Square }\end{array}$ & \multicolumn{2}{c}{$\begin{array}{c}\text { Std. Error of the } \\
\text { Estimate }\end{array}$} \\
\hline 1 &, $718(\mathrm{a})$ &, 515 &, 508 & 1,621 \\
\hline
\end{tabular}

a. Predictors : (Constant), Entrepreneurship leadership

b. Dependent Variable: Job Satisfaction

Based on the results of the data analysis above, the $\mathrm{R}$ correlation coefficient value is 0.718 . This means that there is a good influence between entrepreneurial leadership $\left(\mathrm{X}_{1}\right)$ on job satisfaction $(\mathrm{Y})$. While the determinant coefficient $(\mathrm{KD})$ is seen from $\mathrm{R}$ Square $=0.515$. This means that entrepreneurial leadership contribution $\left(\mathrm{X}_{1}\right)$ to job satisfaction $(\mathrm{Y})$ is $51.5 \%$ while the remaining $48.5 \%$ is determined by other factors such as work culture, employee discipline, work environment, etc. That are not examined. 
t-Test

The hypothesis proposed in this study are as follows:

a. Ho : $\mathrm{Q}=0$ There is no positive influence on entrepreneurial leadership on employee job satisfaction

b. Ha : $\mathrm{Q} \neq 0$ There is a positive influence on entrepreneurial leadership on employee job satisfaction Significance level $\alpha=0.05$

$$
t_{\text {Count }}=\pi r^{2}=\frac{s}{s b} t_{\text {Count }}=4.649
$$

Rejection area:

Ho is rejected if $\mathrm{t}$ count $>\mathrm{t}$ table where $\mathrm{t}$ table $=\alpha ;(\mathrm{n}-2)=\mathrm{t} 0.05 ; 75=0.05 ; 75=1.992$.

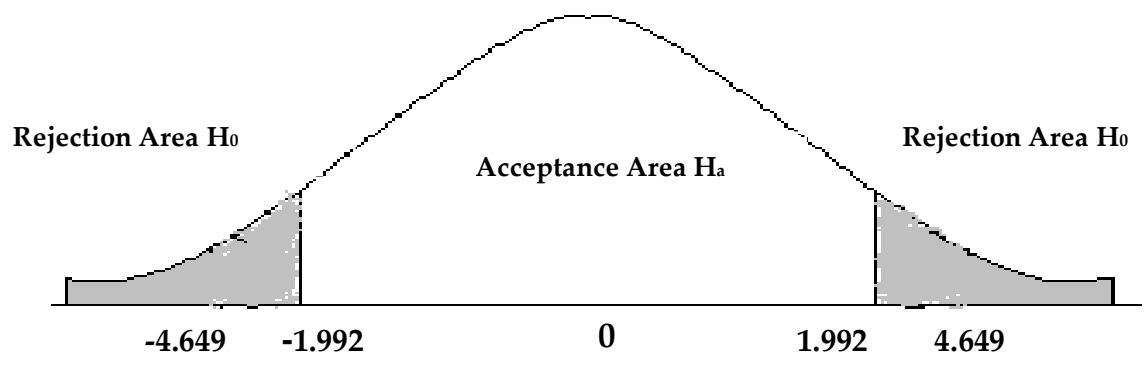

Fig. 4 Decision Area of t-test

Conclusion:

Because $\mathrm{t}$ arithmetic $=4.649>\mathrm{t}$ table $=1.992$ at the significance level of $95 \%$ and $n-2=75$ then, Ho is rejected, which means that there is an influence of entrepreneurial leadership on job satisfaction of employees at Serang Raya University.

Effect of Motivation (X2) on Job Satisfaction (Y)

Regression Test

Table 12 Multiple Linear Regression Coefficients ${ }^{a}$

\begin{tabular}{crrrrrr}
\hline \multirow{2}{*}{ Model } & \multicolumn{2}{c}{$\begin{array}{c}\text { Unstandardized } \\
\text { Coefficients }\end{array}$} & $\begin{array}{c}\text { Standardized } \\
\text { Coefficients }\end{array}$ & T & Sig. \\
\cline { 2 - 7 } & B & Std. Error & Beta & B & $\begin{array}{c}\text { Std. } \\
\text { Error }\end{array}$ \\
\hline 1 (Constant) & 36,964 & 2,743 & & 13,474 &, 000 \\
Motivation &, 200 &, 061 & &, 354 & 3,274 &, 002 \\
\hline
\end{tabular}

a. Dependent Variable: Job Satisfaction

Information:

$\mathrm{Y}$ : Motivation $\left(\mathrm{X}_{2}\right)$

a $\quad$ : 36.964

$\mathrm{bX}_{1}: 0.200$

So it is stated in the form of the regression equation as follows: $Y=a+b X_{2}$

$\mathrm{Y}=36.964+0.200 \mathrm{X}_{2}$ 
Coefficient of Determination (KD)

Table 13 Coefficient of Correlation Between Motivation Against Job Satisfaction

\begin{tabular}{llrrrr}
\hline Model & $\mathbf{R}$ & $\begin{array}{c}\mathbf{R} \\
\text { Square }\end{array}$ & $\begin{array}{c}\text { Adjusted R } \\
\text { Square }\end{array}$ & \multicolumn{2}{c}{$\begin{array}{c}\text { Std. Error of the } \\
\text { Estimate }\end{array}$} \\
\hline 1 &, $354(\mathrm{a})$ &, 125 &, 113 & & 2,117 \\
\hline
\end{tabular}

a. Predictors : (Constant), Motivation

b. Dependent Variable: Job Satisfaction

Based on the results of the data analysis above, the $\mathrm{R}$ correlation coefficient value is 0.354 . This means that there is a moderate influence between motivation $\left(\mathrm{X}_{2}\right)$ on job satisfaction $(\mathrm{Y})$. While the determinant coefficient $(\mathrm{KD})$ seen from $\mathrm{R}$ Square $=0.125$. This means that the contribution of motivation $\left(\mathrm{X}_{1}\right)$ to job satisfaction $(\mathrm{Y})$ is $12.5 \%$ while the remaining $87.5 \%$ is determined by other factors such as work culture, employee discipline.

t-Test

The hypothesis proposed in this study are as follows:

a. Ho : $Q=0$ There is no positive effect of motivation on employee job satisfaction

b. Ha : $\mathrm{Q} \neq 0$ There is a positive effect of motivation on employee job satisfaction

Significance level $\alpha=0.05$

$$
t_{\text {Count }}=\pi r^{2}=\frac{s}{s b} t_{\text {count }}=13.474
$$

Rejection area:

Ho is rejected if $\mathrm{t}$ count $>\mathrm{t}$ table where $\mathrm{t}$ table $=\alpha ;(\mathrm{n}-2)=\mathrm{t} 0.05 ; 75=0.05 ; 75=1.992$.

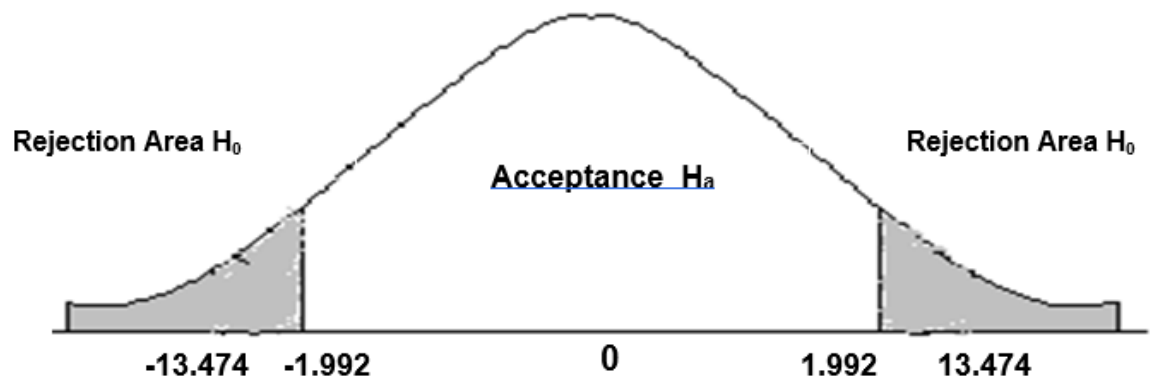

Fig. 5 Decision Area t-test

Conclusion:

Because $t$ count $=13.474>t$ table $=1.992$ at the significance level of $95 \%$ and $n-2=75$ then, Ho is rejected, which means there is an effect of motivation on employee job satisfaction at Serang Raya University. The above analysis shows that the results of hypothesis testing indicate that motivation has a positive influence on job satisfaction, the effect of motivation on employee job satisfaction at Serang Raya University, namely with a correlation coefficient of 0.125 with $\alpha=0.05$, thus contributing motivation variables to employee job satisfaction at The University Attacked 15.5\%. 
The Effect of Entrepreneurship Leadership (X1) and Motivation (X2) on Job Satisfaction (Y) Regression Test

Table 14 Multiple Linear Regression Coefficients ${ }^{a}$

\begin{tabular}{rrrrrrr}
\hline \multirow{2}{*}{ Model } & \multicolumn{2}{c}{$\begin{array}{c}\text { Unstandardized } \\
\text { Coefficients }\end{array}$} & $\begin{array}{c}\text { Standardized } \\
\text { Coefficients }\end{array}$ & T & Sig. \\
\cline { 3 - 7 } & & B & $\begin{array}{c}\text { Std. } \\
\text { Error }\end{array}$ & Beta & \multirow{2}{*}{ B } & $\begin{array}{c}\text { Std. } \\
\text { Error }\end{array}$ \\
\hline \multirow{2}{*}{1} & (Constant) & 14,885 & 3,477 & & 4,281 &, 000 \\
& Leadership &, 614 &, 078 &, 682 & 7,844 &, 000 \\
& Motivation &, 053 &, 049 &, 093 & 1.068 &, 289 \\
\hline
\end{tabular}

Dependent Variable: Job Satisfaction

Information :

Y : Job Satisfaction

a $\quad: 14.885$

$\mathrm{bX}_{1}: 0.614$

$\mathrm{bX}_{2}: 0.053$

So that it is expressed in the form of a regression equation as follows:

$\mathrm{Y}=\mathrm{a}+\mathrm{b} \mathrm{X}_{1}+\mathrm{b} \mathrm{X}_{2}$

$\mathrm{Y}=14.885+0.614 \mathrm{X}_{1}+0.053 \mathrm{X}_{2}$

From the results of the regression equation above shows that entrepreneurial leadership $\left(X_{1}\right)$ value is 0 (zero) then job satisfaction $(\mathrm{Y})$ is 14,885 While the leadership regression coefficient $\left(\mathrm{X}_{1}\right)$ of 0.614 shows that each addition of one entrepreneurial leadership unit will increase job satisfaction by 14,885 . If motivation $\left(\mathrm{X}_{2}\right)$ is 0 (zero) then job satisfaction $(\mathrm{Y})$ is 14,885 while the motivation coefficient $\left(\mathrm{X}_{2}\right)$ is 0.053 indicating that each addition of one unit of entrepreneurial leadership will increase job satisfaction (Y) by 14,885 .

Coefficient of Determination (KD)

Table 15 Correlation Coefficients Between Entrepreneurship Leadership and Motivation

\begin{tabular}{llrrrr}
\multicolumn{5}{c}{ for Job Satisfaction } \\
\hline Model & $\mathbf{R}$ & $\begin{array}{c}\mathbf{R} \\
\text { Square }\end{array}$ & $\begin{array}{c}\text { Adjusted R } \\
\text { Square }\end{array}$ & $\begin{array}{c}\text { Std. Error of the } \\
\text { Estimate }\end{array}$ \\
\hline 1 &, 723 (a) &, 522 &, 509 & & 1,620
\end{tabular}

a. Predictors : (Constant), Leadership, Motivation

b. Dependent Variable: Job Satisfaction

t-Test

The hypothesis proposed in this study are as follows:

a. Ho : $\varrho=0$ There is no positive influence on entrepreneurial leadership and motivation on employee job satisfaction

b. Ha : $\varrho \neq 0$ There is a positive influence on entrepreneurial leadership and motivation on employee job satisfaction

$$
t_{\text {Count }}=\pi r^{2}=\frac{s}{s b} t_{\text {Count }}=4.281
$$

Ho is rejected if $\mathrm{t}_{\text {count }}>\mathrm{t}$ table where $\mathrm{t}$ table $=\alpha ;(\mathrm{n}-2)=\mathrm{t} 0.05 ; 75=0.05 ; 75=1.992$. 


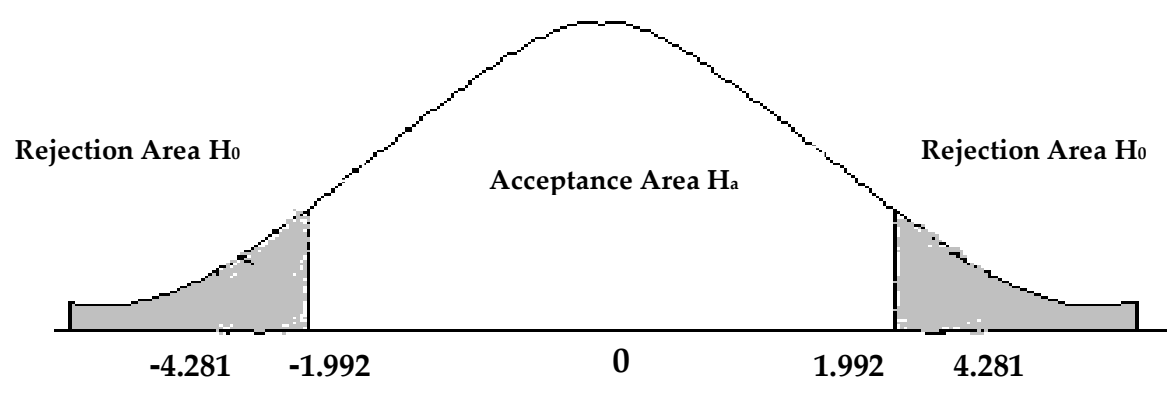

Fig. 6 Decision Area t-test

Because of $n-2=75$ then, Ho is rejected, which means there is an influence of entrepreneurial leadership and motivation on employee job satisfaction at Serang Raya University.

The results of hypothesis testing indicate that entrepreneurial leadership and motivation have a positive influence on employee job satisfaction, the influence of entrepreneurial leadership and motivation on employee job satisfaction at Serang Raya University, namely with a correlation coefficient of 0.552 with $\alpha=0.05$, thus contributing entrepreneurial leadership variables and motivation towards Employee job satisfaction at Serang Raya University is 55.2\%.

D. Hypothesis Test (F Test)

Table 16 F Test Statistics Anova (b)

\begin{tabular}{|c|c|c|c|c|c|}
\hline Model & $\begin{array}{l}\text { Sum of } \\
\text { Squares }\end{array}$ & df & Mean Square & $\mathbf{F}$ & Sig. \\
\hline 1 Regression & 212.222 & 2 & 106.111 & 40.446 & $.000(a)$ \\
\hline Residual & 194.141 & 74 & 2.624 & & \\
\hline Total & 406.364 & 76 & & & \\
\hline
\end{tabular}

a. Predictors : (Constant), Leadership, Motivation

b. Dependent Variable: Job Satisfaction

Testing Criteria:

Ho is accepted if $\mathrm{F}$ count $\leq \mathrm{F}$ table

Ho is rejected if $F$ count $\geq f$ table

Based on the table above it can be seen that the value of $\mathrm{F}$ count is 46,865 , the value is then compared with $\mathrm{F}$ table. If the level of significance is $\alpha=5 \%$, df numerator $(\mathrm{k}-1)$ or $(\mathrm{n}-\mathrm{k})$ or $(77-2)=75$. Then it can be obtained F table value of 3.12 .

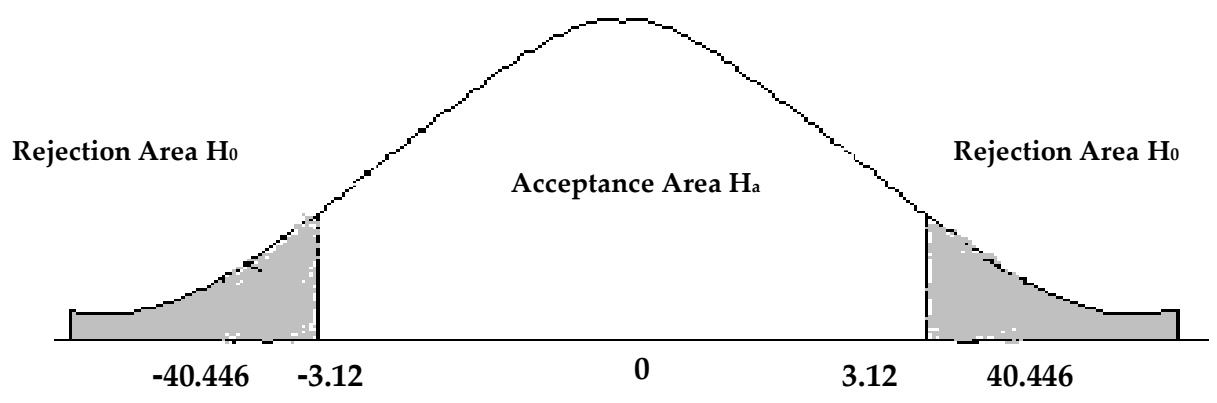

Fig. 7 Decision Area t-test 
From the picture above shows the value of $F$ calculated is greater than $F$ table $(40,446>3.12)$ so that Ho is rejected and Ha is accepted. This means that there is an influence of entrepreneurial leadership and motivation on employee job satisfaction at Serang Raya University.

\section{Conclusions and Suggestions}

Based on the results of research and discussion, a conclusion can be taken as follows:

1. Based on the recapitulation of the average interpretation of $4.68 \%$ and that number includes very good assessment criteria. This means that entrepreneurial leadership has been implemented well even though it is still not optimal. Based on the recapitulation of the average interpretation of $4.43 \%$ and this number includes very good assessment criteria. This means that motivation has been implemented very well even though it is still not optimal. Based on the recapitulation of the average interpretation of $4.59 \%$ and this number includes very good assessment criteria. This means that job satisfaction has been obtained by employees very well even though it is still not optimal.

2. Based on the correlation analysis between entrepreneurial leadership and motivation for job satisfaction obtained at 0.723 Means that there is a good influence of entrepreneurial leadership and motivation on job satisfaction. Furthermore, to find out the size of the contribution of entrepreneurial leadership variables (X1) and motivation (X2) to the variable job satisfaction $(\mathrm{Y})$ used the coefficient of determination with a acquisition value of $52.2 \%$ entrepreneurial leadership and motivation have an influence on job satisfaction. While the remaining $47.8 \%$ is influenced by other factors not examined in this study. Simple linear regression is obtained by the equation $\mathrm{Y}=14.885+0.614 \mathrm{X} 1+0.053 \mathrm{X} 2$, if entrepreneurial leadership (X1) and motivation $(\mathrm{X} 2)$ the value is 0 (zero) then job satisfaction $(\mathrm{Y})$ is 14,885 . While the regression coefficient of 0.614 and 0.053 shows that each addition of one unit of entrepreneurial leadership (X1) and motivation $(\mathrm{X} 2)$ will increase job satisfaction $(\mathrm{Y})$ by 14,885 . Calculations from the test $\mathrm{H} 0$ are rejected and Ha is accepted.

\section{Sugestions}

Based on the description of the conclusions above, it can provide some suggestions that hopefully can contribute to increasing job satisfaction of employees at Serang Raya University as follows:

1. Given that entrepreneurial leadership is very influential on employee job satisfaction, the leadership should create a different atmosphere and pay attention to employees related to the work of employees, assignment of tasks that have been charged to employees, treatment of leaders towards employees, and work environment.

2. Every employee is expected to carry out their duties professionally and responsibly in accordance with their respective duties in order to improve their job satisfaction.

\section{References}

Arep, Ishak, dkk.2003, Manajemen Sumber Daya Manusia.. Universitas Trisakti. Jakarta

Arikunto, Suharsimi. 2002 Prosedur Penelitian. Rineka Cipta. Jakarta

As'ad. 2004, Kepemimpinan dan Motivasi. Ghalia Indonesia. Jakarta.

Budiardjo, Miriam, 2003. Dasar-Dasar Ilmu Politik, Jakarta

Daft Richard L. 2007. Management-Manajemen. Edisi 6 Buku 1. Salemba Empat. Jakarta.

Ghozali, Imam. 2007. Aplikasi Analisis Multivariate Dengan Program SPSS. BP-Universitas Diponogoro, Semarang. 
Greenberg, Jerald dan Baron, Robert A. 2000. Perilaku Organisasi. Jakarta : Prentice Hall.

Handayaningrat, Soewarno, 2002, Manajemen Personalia dan Sumber Daya Manusia, Cetakan pertama, Liberti. Jakarta

Hasibuan, Malayu, S.P. 2003. Manajemen Dasar, Pengertian dan Masalah. Jakarta : PT Toko Gunung Agung.

Karyadi, M., 2001. Kepemimpinan, Karya Nusantara. Bandung

Labovitz, Sanford dan Robert Hagedorn, 2000. Metode Riset Sosial, Erlangga, Jakarta

Naniek Utami Handayani, dkk. (2018). Faktor Penentu Kesadaran Konsumen Dalam Pembelian Produk Kertas Bertanda Eco-Label. Jurnal MIX: Jurnal Ilmiah Manajemen, Volume 8, No. 3, Okt 2018. p. $477 \quad-$ 503. ISSN : 2088-1231. E-ISSN: 2460-5328. DOI: dx.doi.org/10.22441/mix.2018.v8i3.003

Nawawi, Hadari. 2005. Manajemen Sumber Daya Manusia. Gajah Mada University Press. Yogyakarta

Riggio, Ronald E. 2000.Manajemen Kinerja Gramedia Pustaka Utama. Jakarta

Robbin, Stephen P, 2003. Perilaku Organisasi, Edisi Kesembilan, Jilid 2, PT. Indeks Kelompok Gramedia, Jakarta.

Santoso, Singgih. 2007. Statistik Multivariat, PT Gramedia. Jakarta

Siagian, Sondang P., 1992 b, Peranan Staf Dalam Management, Bandung : Gunung Agung.

Sondang P. Siagian, 2010. Teori \& Praktek Kepemimpinan. PT Rieneka Cipta, Jakarta

Sopiah, 2008, Perilaku Organisasional, C.V Andi Offset. Yogyakarta

Sugiyono. 2007 Metode Penelitian Administrasi, CV Alfabeta. Bandung

Supranto, John, 2000. Statistik-Teori dan Aplikasi, Erlangga. Jakarta

Susanto A.B; Koesnadi Kardi, 2003. Pemimpinan dan Kepemimpinan.Raja. Grafindo Persada. Jakarta.

Sutrisno, Edy. 2010. Manajemen Sumber Daya Manusia; PT Prenada Media. Group. Jakarta

Sutrisno Hadi, 2004. Statistik Jilid 2, Andi Offset. Yogyakarta

Tangkilisan, H.N.S., 2005, Manajemen Publik, Jakarta : Grasindo

Thoha, Miftah. 2003. Perspektif Perilaku Birokrasi. PT. Raja Grafindo Persada. Jakarta

Wibowo. 2010. Manajemen Kinerja. PT. Rajagrafindo Persada, Jakarta.

Yatim Riyanto, 2001 Metodologi Penelitian Pendidikan, SIC Sura. 